\title{
An unusual cause of arthritis
}

\author{
K. D. MORLEY* \\ M.R.C.P., F.R.A.C.P.
}

H. R. VICKERS†

M.D., M.Sc., F.R.C.P.

\author{
G. R. V. HUGHES \\ M.D., F.R.C.P.
}

Rheumatology Unit and †Dermatology Department, Hammersmith Hospital and Royal Postgraduate Medical School, Du Cane Road, London W12

\section{Summary}

We present a case of leprosy, an uncommon infection in the United Kingdom, which mimicked features of widely known rheumatic diseases and resulted in subsequent delay of diagnosis.

KEY WORDS: borderline tuberculoid leprosy, arthritis, neuropathy.

\section{Case report}

A 39-year-old Indian lady presented to the rheumatology outpatient clinic with a 6-month history of pain, stiffness and swelling in several fingers, wrists, knees and feet. She had previously been examined elsewhere and prescribed prednisolone initially in high dose, for an undiagnosed arthritis. For 3 months, she had recurrent blistering lesions on the extensor surfaces of several fingers and the palms of her hands in the region of the metacarpo-phalangeal (MCP) joints, each of which lasted for a few days. She noted that her hands were a bluish colour at times, but there was no clear history of Raynaud's phenomenon. During the previous 6 weeks, she had developed a macular scaly rash, which resembled psoriasis, on the back of her hands, extensor surface of the arms, knees, lower legs and feet (Fig. 1). There were no significant constitutional symptoms. She had lived in England for 8 years apart from a 3 month visit to Central India 2 years before presentation. There was no family history of arthritis. On examination, she had diffuse swelling of the wrists and MCP joints of both hands. There were small effusions in both knees.

Investigations revealed normal haematology, erythrocyte sedimentation rate, electrolytes, uric acid and hepatic enzymes. The latex rheumatoid factor test was positive on several occasions up to $1 / 160$, but IVB.

*Present address i Ninewells Hospital, Ninewells, Dundee DD2 also negative on several occasions before treatmente Antinuclear antibody was negative and no antibodies to extractable nuclear antigens were found. IgG was slightly raised. Radiology of the hands and wris showed periarticular osteoporosis and probably smadl erosions in the heads of one metacarpal and 3 metatarsal bones.

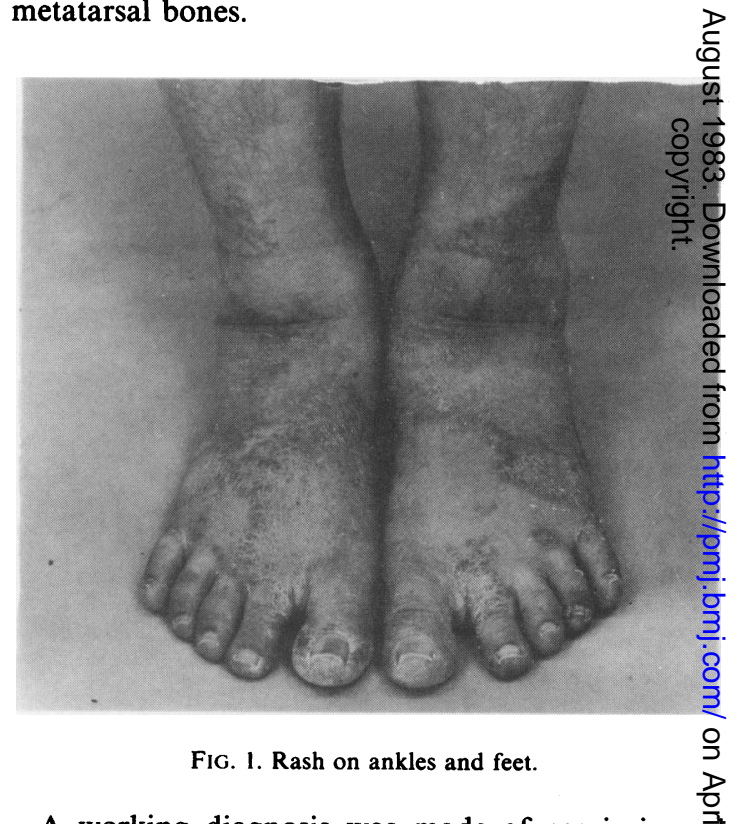

A working diagnosis was made of psoriasis and associated arthritis. She was treated with now steroidal anti-inflammatory drugs and local steroind injections. The dose of prednisolone was slow reduced. Her arthritis symptoms improved during the subsequent few weeks but she continued to have recurrent blistering lesions on her hands, and the rash on her arms and feet persisted.

Two months after presentation to the rheumato logy unit, she first complained of numbness and 
tingling on both hands. It was evident that she had a sensory neuropathy and also wasting of the small muscles in her hands. The previous scaling eruption gradually became more indurated and an oval, welldefined lesion appeared on the right side of the chin. This lesion was noted to be anaesthetic. A clinical diagnosis of leprosy was made. Skin biopsies were performed from the rash on her right foot and chin and granulomata were seen containing Mycobacteria leprae. The histological classification was borderline tuberculoid leprosy. Treatment began with dapsone, rifampicin and prednisolone (WHO Report, 1982). Six months later, there was resolution of the rash but residual neurological damage with ulnar and median nerve clawing of her hands (Fig. 2) for which she may require tendon transplants.

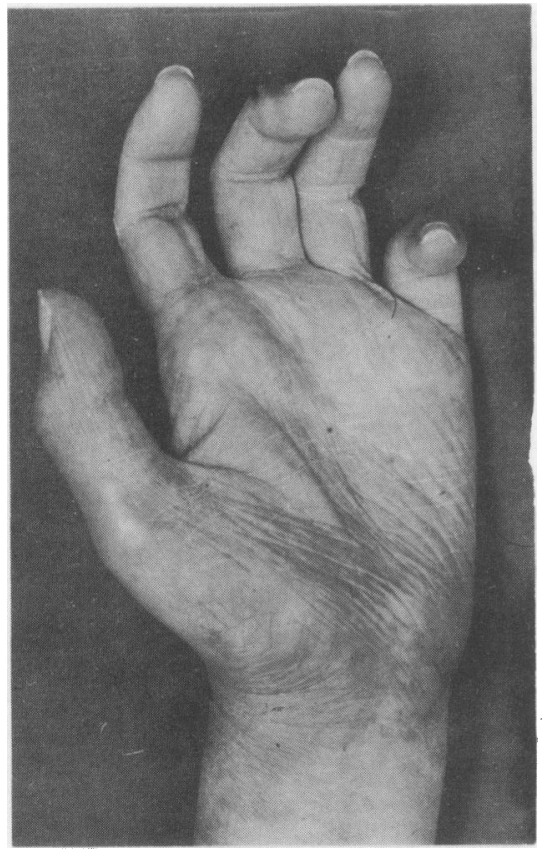

FiG. 2. Small-muscle wasting and clawing of hands.

\section{Discussion}

It is estimated that $12-20$ million people suffer from leprosy worldwide but the disease is relatively rare in the United Kingdom with 23 notified new cases in England and Wales during 1981 and 353 patients registered as receiving treatment. All of these patients are thought to have contracted the infection when abroad. We assume that our patient acquired the disease in Central India, an area where leprosy is endemic, before she came to live in this country.

The disease displays a clinico-pathological spectrum related to the host-ability to develop and sustain specific cell-mediated immunity. The 2 polar forms are low resistance 'lepromatous' leprosy, which is a generalized disease, with many organisms in the widespread lesions and high resistance, and 'tuberculoid' leprosy, where localized signs are restricted to skin and nerves. The unstable intermediate part of the range is called 'borderline' and this form untreated tends to downgrade towards the lepromatous end of the spectrum or with effective treatment may undergo a 'reversal' reaction with increasing ability of the patient to produce delayed hypersensitivity and contain the infection.

Rheumatic manifestations of leprosy have been recorded previously (Albert, Weisman and Kaplan, 1980; Karat et al., 1967; Berman, 1979) and there is mention of stiff joints as a complication of leprosy in ancient Chinese literature (Mesner, 1979). These rheumatic symptoms may be an important cause of continuing morbidity in leprosy (Albert et al., 1980).

In borderline tuberculoid (BT) leprosy, the skin and nerve lesions are the primary abnormalities. Inflammation associated with active and reactive BT lesions may cause usually asymmetrical swelling in the hands and feet, particularly if the skin lesions are present at these sites. We think that this was the nature of our patient's rheumatological problems.

Arthralgia is a recognized clinical manifestation of 'reaction' in leprosy (Job, Gude and Macaden, 1964). A true arthritis may occur particularly in a erythema nodosum leprosum (ENL) which is a reactional state of lepromatous leprosy. Karat et al. (1967) reported a rheumatoid arthritis-like syndrome in 10 patients with ENL. The principally affected joints were hands, wrists, knees and ankles. The IgM rheumatoid factor was weakly positive in 6 of the 10 patients, but there were no erosions found on X-ray. Synovial biopsy in 2 cases showed inflammatory changes but no acid-fast bacilli. Albert et al. (1980) found that 15 of 21 patients with leprosy had rheumatic manifestations and these features were the major cause of continuing morbidity. The largest single group was 14 patients with erythema nodosum leprosum of whom 5 had objective evidence of arthritis. They found an exudative inflammatory joint fluid and such was also reported in a case of leprosy by Berman (1979).

The diagnosis in our patient was delayed because the rash, modified by treatment with systemic corticosteroids, resembled psoriasis and her neurological deficit was not apparent until several months after the onset of the other symptoms. The blistering lesions were thought to be due to local neuropathic trauma. Rheumatologically, she had apparent synovitis affecting the MCP joints and wrists but note was made about the rather unusual diffuse thickening in her hands. Radiologically, her hands and wrists showed changes compatible with early inflammatory 
arthritis. The fluctuation of IgM rheumatoid factor titres contributed to the diagnostic difficulty particularly when psoriatic arthritis was being considered. The diagnosis was made clinically when she developed the neurological signs, the skin lesions became less scaling and more indurated and the annular anaesthetic patch appeared on the chin. An important clinical sign noted at a late stage was thickening of her ulnar and superficial radial nerves.

\section{Conclusion}

This case demonstrates an unusual cause of arthritis. Mycobacterial infections are often considered in disease affecting the immigrant population of this country but leprosy is diagnosed only rarely. The possibility of leprosy should be considered in any patient who has lived in an endemic area and develops a persistent rash, unusual arthritis or unusual peripheral neuropathy. The clinician should test skin lesions for sensation, especially light touch and pinprick and feel for enlarged peripheral nerves particularly superficial radial, ulnar and lateral popli- teal. In addition, biopsy of active skin lesions shouf be performed. Clinical diagnosis in this case w\$ delayed because the rash was modified by systemæc steroids. Her skin and joint disease have responded well to appropriate treatment, but she has permane $\overrightarrow{\Rightarrow t}$ neurological damage, some of which might have been avoided if the diagnosis had been made earlief

\section{References}

Albert, D.A., Weisman, M.H. \& KaPlan, R. (1980) The rheumati manifestations of leprosy. Medicine (Baltimore), 59, 442.

Berman, L. (1979) Exudative arthritis in leprosy. Arthritis Rheumatism, 22, 593.

JoB, C.K., GUDE, S. \& MACADEN, V.P. (1964) Erythema nodosum leprosum. A clinicopathological study. International Journal Leprosy, 32, 177.

Karat, A.B.A., Karat, S., Job, C.K. \& Furness, M.A. (196\%) Acute exudative arthritis in leprosy-rheumatoid-arthritis-like syndrome in association with erythema nodosum leprosum. British Medical Journal, 3, 770.

MESSNER, R.P. (1979) Arthritis due to mycobacteria and fungi. Arthritis and allied conditions (Ed. D. McCarthy), p. 1380. Liep Febiger, Philadelphia.

WORLD HEALTH ORGaNiZation (1982) Technical report series AO 675.

(Accepted 25 November 1982) 